\title{
Intelligent control framework for biomass drying process with flue gases based on FLC and CAN bus
}

\author{
Jin Sun ${ }^{\mathrm{a},{ }^{*}}$, Yongan $\mathrm{Xu}^{\mathrm{a}}$, Xionglong Zhu ${ }^{\mathrm{a}}$, Wangqin Zhang ${ }^{\mathrm{b}}$, Xiaowei $\mathrm{Cao}^{\mathrm{a}}$ \\ a Yangzhou University, 196\# West Huayang Road, Yangzhou 225127, China \\ b Yangzhou First People's Hospital, 368\# Hangjiang Mid Road, Yangzhou 225001, China \\ *Corresponding Author: sunjin1001@126.com
}

\begin{abstract}
This paper proposes an intelligent control framework for biomass drying process with flue gases based on FLC (fuzzy logic controller) and CAN (Controller Area Network) bus. In the operation of a biomass drying process, in order to get the biomass with the set-point low moisture content dried by waste high temperature flue gases, it is necessary to intelligent control for the biomass flow rate. Use of an experiment with varied materials at different initial moisture contents enables acquisition of the biomass flow rates as initial setting values. Set the error between actual straw moisture content and set-point, and rate of change of error as two inputs. the biomass flow rate can be acquired by the fuzzy logic computing as the output. Since the length of dryer is more than twenty meters, the integration by the CAN bus can ensure real-time reliable data acquisition and processing.

The control framework for biomass drying process can be applied to a variety of biomass, such as, cotton stalk, corn stalk, rice straw, wheat straw, sugar cane. It has strong potential for practical applications because of its advantages on intelligent providing the set-point low moisture content of biomass feedstock for power generation equipment.
\end{abstract}

Keywords: Intelligent control framework, fuzzy logic controller, Controller Area Network.

\section{Introduction}

As a form of renewable energy, the biomass can be dried by dryers and then be provided as the input fuel for a subsequent power plant through thermo-chemical treatments, such as combustion or gasification. According to the drying medium, the dryers can be classified flue gas dryers and superheated steam dryers, etc. ${ }^{(1)}$; The most common dryer types are belt dryers, rotary dryers, flash dryers and fluidized bed dryers. Belt dryers have the widest range of applications. Professor $\mathrm{Li}^{(2)}$ drew the conclusion that the belt dryers are better suited to take advantage of low-grade and waste heat since they operate at lower temperatures with reduced fire risks, fewer gaseous emission and low energy consumption.

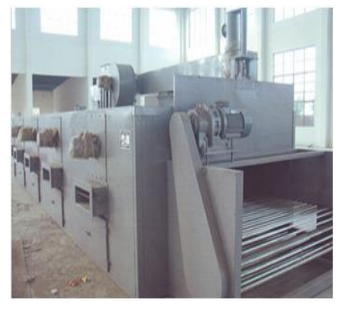

(a) Belt dryer

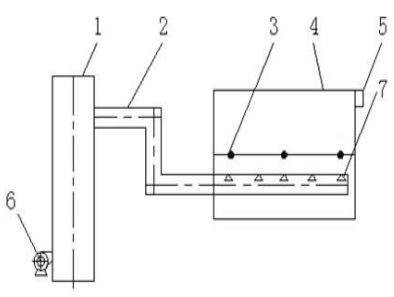

(b) schematic diagram
1.Flue gas; 2. pipeline; 3.temperature test point; 4.belt dryer;

5.fan; 6. hot air blower; 7.control value

Fig. 1. Belt dryer and its schematic diagram

As shown by Fig. 1. is the belt dryer and its schematic diagram. In the belt dryer, the feedstock was spread on a moving perforated conveyor to dry the material in a continuous process. Fans blow the drying medium through the belt and biomass material. Water can be removed from the surface of the biomass, because the temperature of the flue gas is higher than that of the biomass. After drying, the moist air is discharged from the rear through duct by a centrifugal blower. Then the dried biomass was transfer into the down storage for combusting. The biomass feed stocks are mostly wet and need to be dried from nearly $60 \%$ to $15 \%$. Thus the drying is an energy intensive process in the industrial energy utilization ${ }^{(3)}$. In the operation of a biomass drying process, the final goal is to get the biomass of the set-point low moisture content. However, when the biomass flow rate was controlled by manual, the temperature of the 
flue gas was given and the initial moisture content of the biomass was different, the final moisture content cannot mostly reach at the set-point, affecting the efficiency of the following combustion seriously. Consequently, it is necessary to intelligent control for the biomass flow rate to get the biomass of the set-point low moisture content.

To address these problems, some new methods have been developed. In the year of 2005,Alvarez-López et $a l{ }^{(4)}$ considered the error between relativity humidity reference and actual relativity humidity, and the derivative of the error as input, the inlet air as output ,then designed the control system based on fuzzy logic for tobacco drying. In 2006, Atthajariyakul et al. ${ }^{(5)}$ used adaptive fuzzy method in a fluidized bed paddy dryer to control two parameters: air flow and temperature. Mansor et al. ${ }^{(6)}$ presented an application of fuzzy logic controller for plants seeds dryer in 2009. The controller consisted of two inputs: the error between actual grain moisture content and set-point, and the rate of change of error. There was one fuzzy output: the grain flow rate. The control system can provide very fast response to make the grain output moisture content close to the set-point and to reject the disturbance during the grain drying process. Gianini Regina Luz et al. ${ }^{(7)}$ proposed a dynamic model and designed PID control system in the soybean meal drying of an industrial direct rotary dryer in 2010.Load disturbances as step, pseudorandom, and impulse inputs were applied in the dryer. Then, the output responses for perturbations were investigated with control systems. M.Zeynali et al. ${ }^{(8)}$ analyzed fuzzy logic in designing dryer controller to achieve admissible results in 2011. It had three input variables (temperature, humidity and weight) and two outputs variables (heater and fan voltage). The system was capable of drying agricultural products, foods, industrial products etc. In 2013, Khazaei et $a l .{ }^{(9)}$ developed an artificial neural network to predictive model of the grape drying in a hot air dryer. The inputs were air drying temperature, velocity, shrinkage and moisture content at time and the output was moisture content.

However, as far as we know, the use of intelligent control system for straw drying process has rarely been reported. Since the energy management is an essential part of any drying process ${ }^{(10)}$. Therefore, in this paper, we proposed an intelligent control framework for straw drying process with flue gases, which can get the biomass of the set-point low moisture content based on the technique of FLC and CAN bus. The technique of FLC can adjust the biomass flow rate intelligently to meet the final moisture content. And the technique of CAN bus can ensure real-time reliable data acquisition and processing since the length of dryer is more than twenty meters.

\section{MATERIALS AND METHODS}

\subsection{Experiment of the biomass flow rates for initial setting values}

Test purpose: Analysis of the required drying time when different straw was dried from different initial moisture content to final moisture content of $15 \%$. According to this data, we can set the initial speed of the moving perforated conveyor.

Test equipment: $Z R Y-2 P$ high temperature integrated thermal analyzer.

Test object : Four kinds of crop straw, namely cotton stalk, wheat straw ,corn straw and corn cob. The initial moisture content are set as 25\%, 35\%, 40\%, 55\%, $70 \%$. According to the different suppliers ,the temperature of flue gases are set as $110^{\circ} \mathrm{C}, 120^{\circ} \mathrm{C}, 130^{\circ} \mathrm{C}$.

Test procedure was as following, and the result can be seen in Table.1.

Table 1. Experiment of the biomass flow rates

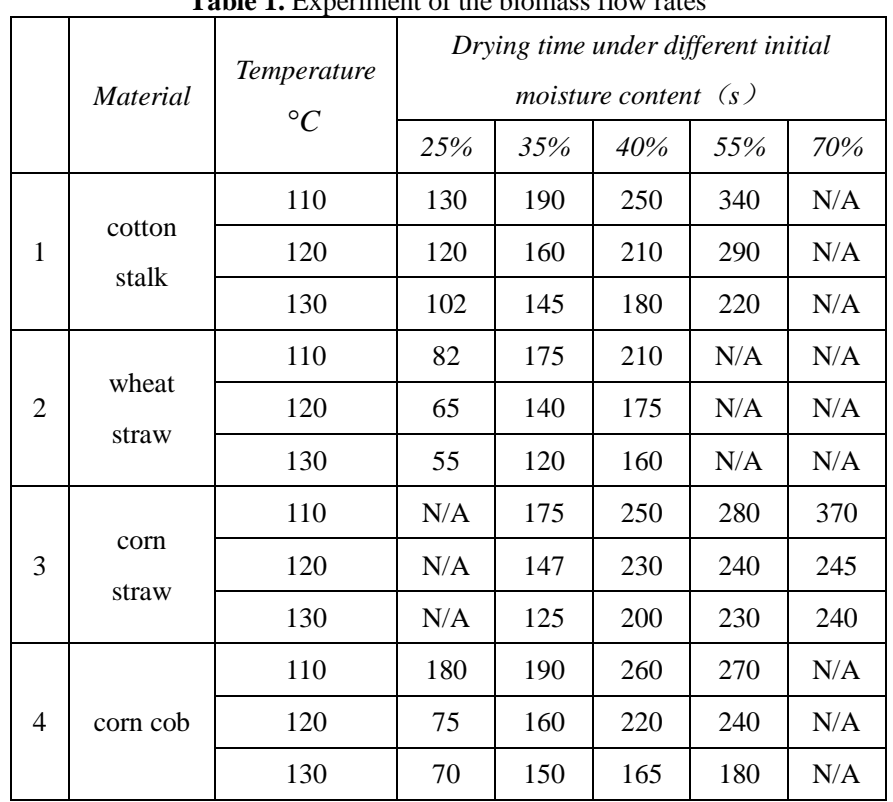

1) Start the computer and open the gas, the gas pressure is maintained at $0.3_{M p a}$. The gas flow is set at 60-80 $\mathrm{ml} / \mathrm{min}$;

2) Set the range of each operating unit and the instrument to zero;

3) Set the temperature control program: Using the maximum heating rate, in the shortest time to reach the set drying temperature, and has maintained this temperature;

4) After the drying temperature is reached,keep the temperature unchanging;

5) Acquisition and conversion of the data: a WordPad 
document can be converted from sample data by the conversion software.

\subsection{Fuzzy logic computing for the output of biomass flow rates}

In this section, the controller designed for this system was described. To design a fuzzy system with mamdani method and center of gravidity defuzzifier, firstly, inputs and output need to be determined, and the membership functions were defined depending on the need for every input and output. Secondly, fuzzy rules were written based on input's and output's membership functions. At last, the surface was achieved for the desirability of system's behavior.

\section{A. Input variables and their membership function}

As shown in Fig. 2, humidity error and weight error were system inputs. For each of their sets, membership functions were defined as triangular and trapezoidal which are good indicators of their sets.

1) Humidity error $H_{e}$

$$
\begin{aligned}
& H_{e}=\left(\Delta H_{s e t}-\Delta H_{a c t}\right) \times 100 \% ; \\
& \Delta H_{s e t}=H_{p r i}-H_{s e t} ; \Delta H_{a c t}=H_{p r i}-H_{a c t}
\end{aligned}
$$

Where $H_{s e t}, H_{p r i}$ and $H_{a c t}$ were humidity set-point, primary humidity and actual final humidity value after dying, respectively. We defined five sets: Negative, Negative small, Zero, Positive small, Positive, where Negative means $H_{a c t}$ is bigger than $H_{\text {set }}$.

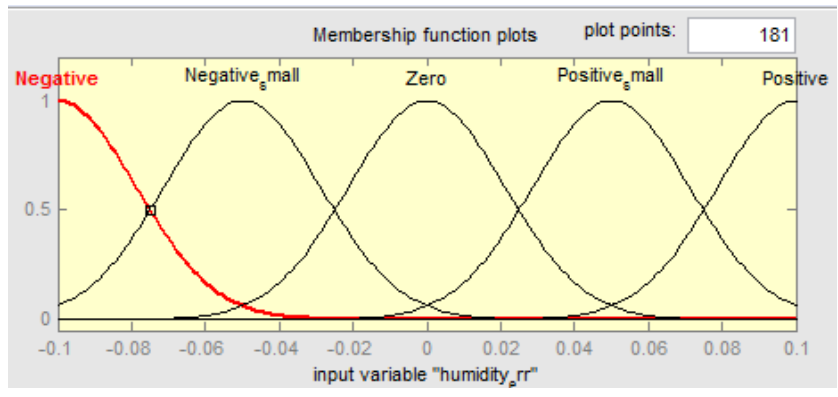

(a) humidity error

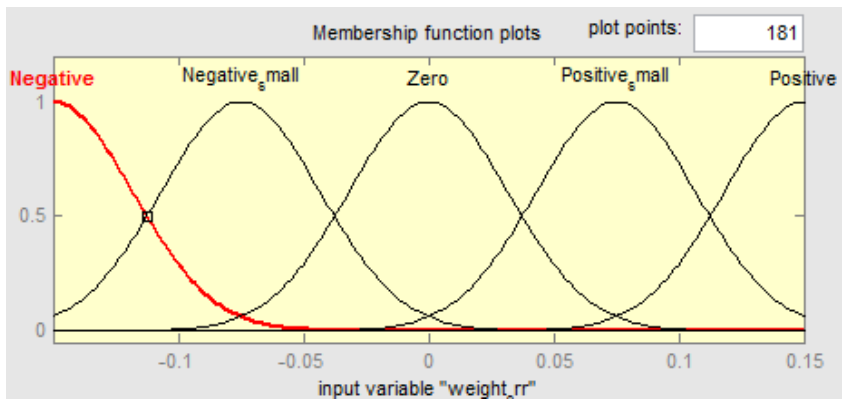

(b) weight error

Fig. 2. The membership function of input variables
2) Weight error $W_{e}$

$$
W_{e}=\frac{W_{p r i}-W_{a c t}}{W_{p r i}} \times 100 \%
$$

Where $W_{p r i}$ was the initial weight and $W_{a c t}$ was the final weight. Weight variable was a good parameter to indicate the amount product drying rate. The weight variable was defined with five sets: Negative, Negative small, Zero, Positive small, Positive, where Zero was a desired weight requested by the user and reaching to this weight meant the end of operation; Negative means the drying rate is too slow and Positive means the drying rate is too quick.

\section{B. Output variable and the membership function}

The output variable was straw flow rate. The seven sets Very slow, Slow, Not so slow, Normal, Not so fast, fast, very fast were defined for straw flow rate. The membership function of Output variable can be seen in Fig. 3.

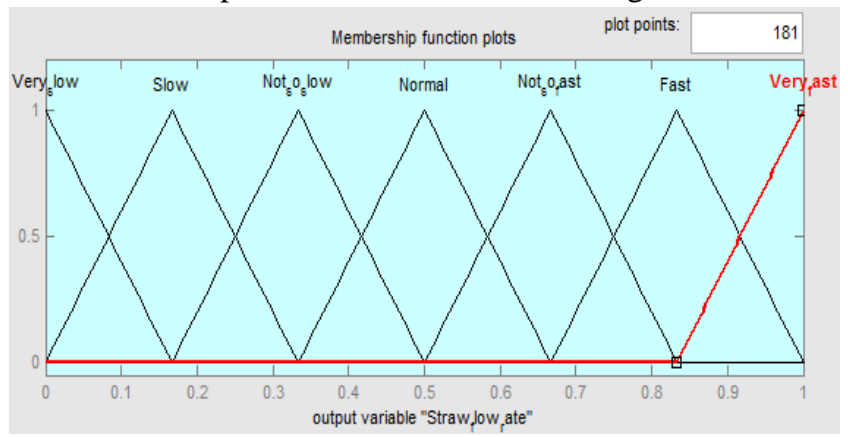

Fig. 3. The membership function of output variable

\section{Fuzzy rules and surface Viewer}

With correct rules, we can expect an appropriate control system. The general form of rules in this article is as follows: IF $<$ weight error is ...> AND $<$ humidity error is ...> THEN < Straw flow rate is ...>. According to membership functions, 25 rules were written in the form of the following

\begin{tabular}{|c|c|c|c|c|c|}
\hline $\mathrm{We}^{\mathrm{he}}$ & Negative & $\begin{array}{c}\text { Negative } \\
\text { small }\end{array}$ & Zero & $\begin{array}{c}\text { Positive } \\
\text { small }\end{array}$ & Positive \\
\hline Negative & Very slow & Very slow & Slow & $\begin{array}{c}\text { Not so } \\
\text { slow }\end{array}$ & Normal \\
\hline $\begin{array}{c}\text { Negative } \\
\text { small }\end{array}$ & Very slow & Slow & $\begin{array}{c}\text { Not so } \\
\text { slow }\end{array}$ & Normal & Not so fast \\
\hline Zero & Slow & Not so slow & Normal & Not so fast & Fast \\
\hline $\begin{array}{c}\text { Positive } \\
\text { small }\end{array}$ & Not so slow & Normal & Not so fast & Fast & Fast \\
\hline Positive & Not so slow & Normal & Not so fast & Very fast & Very fast \\
\hline
\end{tabular}
tables, as shown in Table. 2.

Table 2 Fuzzy rules

Notes. a: humidity error; b: weight error

If humidity error is Negative and weight error is Negative, then straw flow rate is very slow. In Fig. 4., the surface of straw flow rate was plotted based on humidity and weight error. The more smooth the surface was, the 
better the performance of the system was.

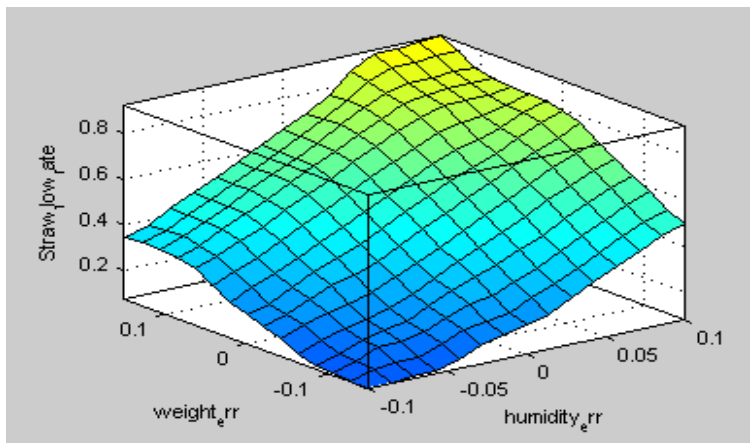

Fig. 4. The surface of straw flow rate based on humidity and weight error

\subsection{CAN bus setting for reliable data acquisition and processing}

As shown in Fig. 5. was CAN bus setting for reliable data acquisition and processing. As soon as we got the signal feedback of humidity, we did the fuzzy logic computing for the output of biomass flow rates, and sent data the drive module to adjust the electric motor to get a suitable biomass flow rates.

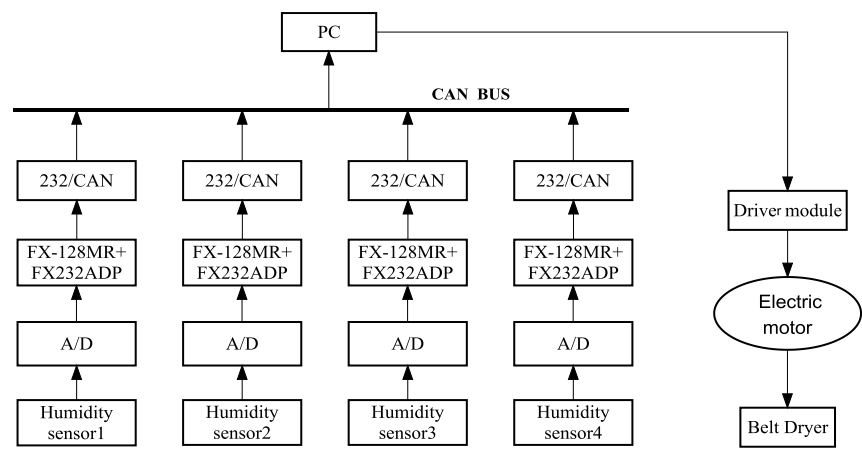

Fig. 5. CAN bus setting for reliable data acquisition and processing

The PLC was Mitsubishi FX series, its format of protocol frame is as following ${ }^{(11)}$ :

\begin{tabular}{|c|c|c|c|c|c|c|}
\hline $\begin{array}{c}\text { Start } \\
\text { (STX) }\end{array}$ & $\begin{array}{c}\text { First } \\
\text { Comman } \\
\mathrm{d}\end{array}$ & $\begin{array}{c}\mathrm{s} \\
\text { of soft } \\
\text { eleme } \\
\mathrm{nt}\end{array}$ & $\begin{array}{c}\text { Byte } \\
\text { coun } \\
\mathrm{t}\end{array}$ & $\begin{array}{c}\text { Data } \\
\text { bloc } \\
\mathrm{k}\end{array}$ & $\begin{array}{c}\text { End } \\
\text { (ETX) }\end{array}$ & $\begin{array}{c}\text { And } \\
\text { verificatio } \\
\mathrm{n}\end{array}$ \\
\end{tabular}

PLC returns the correct response frame format for operating the read data register value ,as follows:

\begin{tabular}{|l|l|l|l|}
\hline Start (STX) & Value & End (ETX) & And verification \\
\hline
\end{tabular}

The CANopen protocol was used as CAN BUS in Fig.5. CANopen is a family of high-level protocols for distributed automation control devices to communicate based on CAN bus. CANopen device is consist of communication protocol, object dictionary and application programs. The communication protocol defines four categories of communication objects: SDO(Service Data Object),PDO (Process Data Object),NMT (Network management) and special function objects. SDO is used to visit the object dictionary, and to configure the communication and control parameters; PDO is used for the transmission of real-time data; NMT is used for the monitoring and management of master node for the passive node; Special functional objects are used to communicate and network management of the above three categories of objects $^{(12)}$.We can visit "application objects" of the device (such as input / output device parameters and functions and network variables, etc.) from the object dictionary entry. The entry of the object dictionary was often determined by an index and sub-index uniquely. The application programs became the bridge between the CANopen device and the PC. PC can be used to configure for SDO devices, and to using high-speed data exchange between PDO and the CANopen devices.CANopen protocol transported the real-time data and service data separately, making real-time data transmission more efficiency. As shown in Fig.5, 232 / CAN gateway allowed each PLC to have the interface connecting with CANopen bus. Each PLC can set a separate device ID number, which length is 11 or 29 , as the address of the PLC unit. In such a network of multiple PLCs composited by unlimited number of PLC serials; And any PLC can initiate the data communications, which will not be not lost, because the gateway can automatically arbitrate the data. By means of the "virtual serial port" software, CANopen network can build up to 2047 standard serial communication ports, which can connect up to 2047 serial network. In this way, we can give full play to the role of industrial PC and get an efficient communication.

\subsection{System Application and Testing}

As shown in Fig. 6.,this system contains two controllers: one was PID controller module, the other was Fuzzy logic controller module. There was a constant module in the left of Switch module. If the parameter in the constant module is set as " 1 ", Fuzzy logic controller module can work in this system, and If the parameter is " 1 ", PID controller can work. The control objects of the system contained straw flow rate module and straw dryer module. By regulate the straw flow rate, the expected straw humidity can be acquired. The Signal Generator module was a square wave, which output amplitude was 0.5 and frequency was $0.1 \mathrm{Rad} / \mathrm{s}$.The Comparison module displayed the comparison between the square wave and the response signal, as shown in Fig. 7. can be seen that the performance of Fuzzy logic controller was better than that of PID controller. The label of horizontal axis is time(which unit is second), while the label of vertical axis is 
wave width(which unit is $\mathrm{mm}$ ).

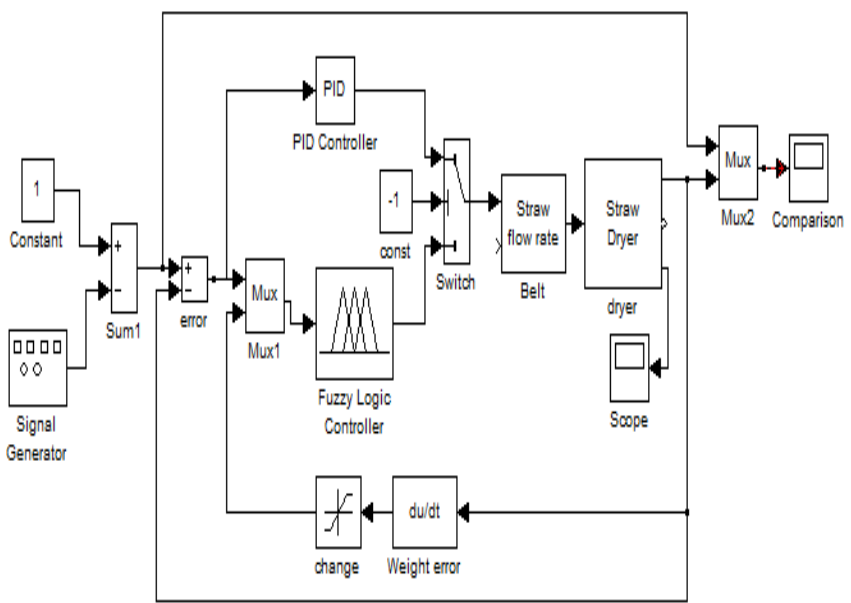

Fig. 6. The system of Fuzzy logic controller and PID controller

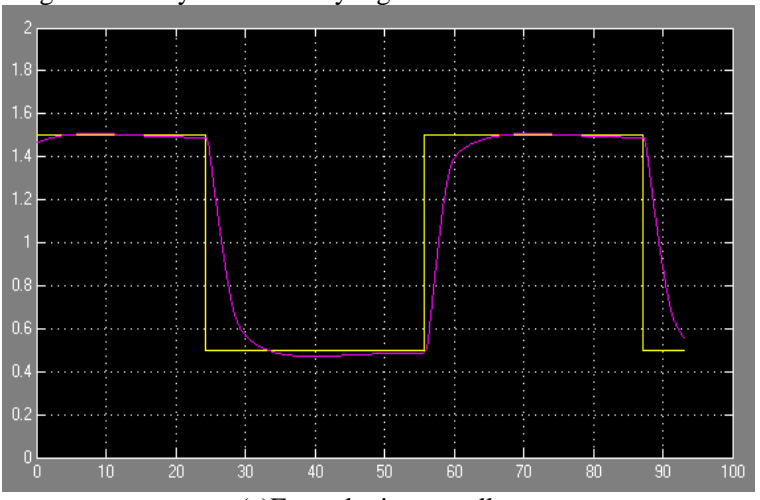

(a)Fuzzy logic controller

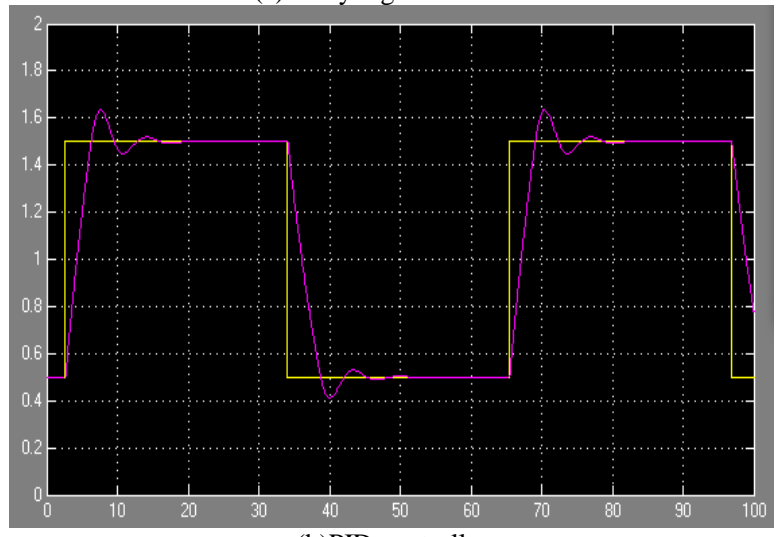

(b)PID controller

Fig. 7. Comparison of fuzzy logic controller and PID controller

The production test site was shown in Fig. 8. Test material was corn straw, and the temperature of flue gases are set as $130{ }^{\circ} \mathrm{C}$. The initial moisture content were set as $40 \%$ and the expected moisture content was $15 \%$.Fig. 9. were the change curves of the straw moisture after drying under the manual control(constant straw flow rate) and the Fuzzy control condition. Under the manual control, the finial moisture content was $17 \%$, while in the Fuzzy control condition, the finial moisture content was $15 \%$, meeting the customer's expectations. Consequently, an intelligent control framework for straw drying process based on FLC and CAN bus was established, and the Fuzzy logic control with the advantages of straw discharge speed optimization, can compensate for changes of straw drying conditions, and was particularly effective for nonlinear and large delay drying control.
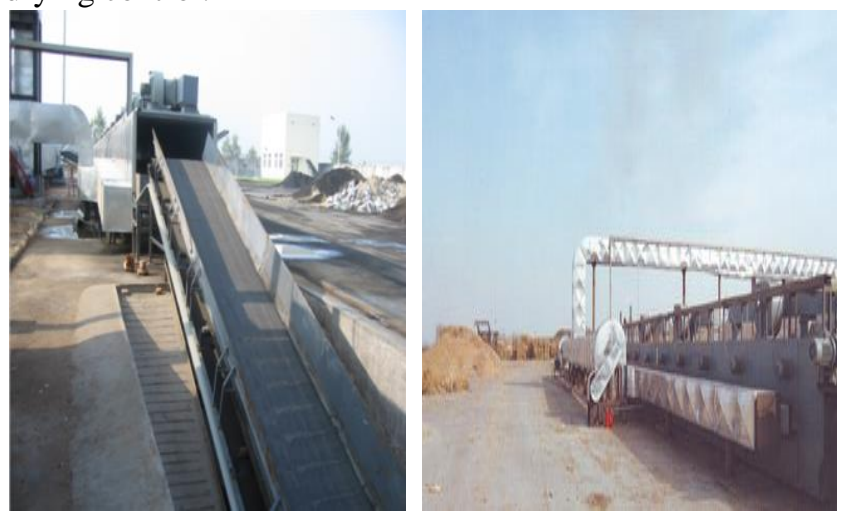

Fig. 8. Test site

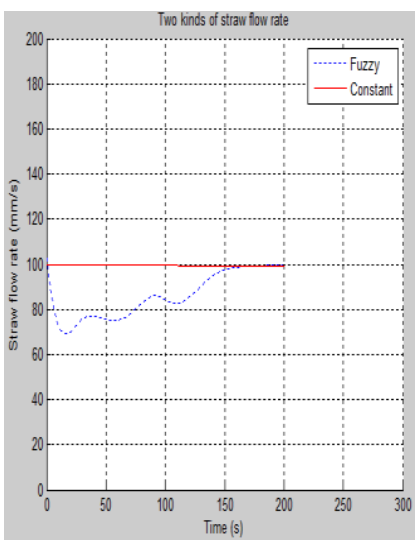

(a)Two kinds of straw flow rate

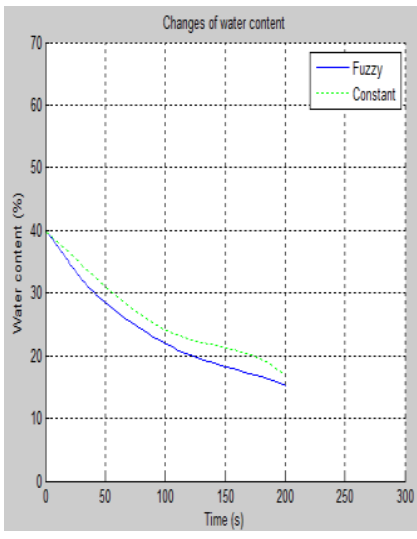

(b)Changes of water content
Fig. 9. Test result

\section{Conclusions}

Dry biomass can provide considerable benefits for power generation. This paper investigated a biomass drying process by belt dryer using low-grade waste heat as the heat source. Biomass drying process has a nonlinear process and multivariable model achieving which necessitates hard work. In order to get the biomass with the set-point low moisture content dried by waste high temperature flue gases, this paper proposed an intelligent control framework for biomass drying process with flue gases based on FLC and CAN bus. Use of an experiment with varied materials at different initial moisture contents enabled the acquisition of the biomass flow rates as initial setting values. Set the error between actual grain moisture content and set-point, and rate of change of error as two inputs. the biomass flow rate can be acquired by the fuzzy logic computing as the output. Since the length of dryer is more than ten meters, the integration by the can bus can ensure real-time reliable data acquisition and processing. The control framework for biomass drying process can be applied to a variety of biomass, such as, cotton stalk, corn stalk, rice straw, wheat 
straw, sugar cane. It has strong potential for practical applications because of its advantages on intelligent providing the set-point low moisture content of biomass feedstock for power generation equipment.

\section{Acknowledgment}

This work was supported by National Natural Science Foundation of China(No. 51475409), Jiangsu Province Basic Research Program Natural Science Foundation (No. BK20141277), China Postdoctoral Science Foundation Funded Project(No. 2013M541736), Jiangsu Planned Projects for Postdoctoral Research Funds(No. 1302179C), and Yangzhou city - Yangzhou University of Science and Technology Cooperation Program Funds (No. 2012038-12).

\section{References}

(1) L. Fagernäs,J. Brammer,C. Wilén,M. Lauer, and F. Verhoeff:" Drying of biomass for second generation synfuel production", Biomass and Bioenergy, Vol. 34, No.9, pp 1267-1277,2010.

(2) H. Li,Q. Chen,X. Zhang,K. N. Finney,V. N. Sharifi, and J. Swithenbank: "Evaluation of a biomass drying process using waste heat from process industries: A case study", Applied Thermal Engineering, No. 35, pp71-80,2012.

(3) K. Chua,A. Mujumdar,M. Hawlader,S. Chou, and J. Ho: "Batch drying of banana pieces-effect of stepwise change in drying air temperature on drying kinetics and product colour", Food Research International, Vol. 34, No.8, pp 721-731, 2001.

(4) I. Alvarez-López,O. Llanes-Santiago, and J. L. Verdegay: "Drying process of tobacco leaves by using a fuzzy controller, Fuzzy sets and systems", Vol. 150, No. 3, pp493-506,2005.

(5) S. Atthajariyakul, and T. Leephakpreeda: "Fluidized bed paddy drying in optimal conditions via adaptive fuzzy logic control", Journal of food engineering, Vol. 75, No. 1, pp104-114,2006.

(6)H. Mansor,S. Noor,R. Ahmad,F. S. Taip, and O. F. Lutfi: "Fuzzy Control of Grain Drying Process, Computer Modelling and Simulation", 2009. UKSIM'09. 11th International Conference on IEEE, pp9-13,2009.

(7)G. R. Luz,W. A. dos Santos Conceição,L. M. de Matos Jorge,P. R. Paraíso, and C. M. G. Andrade: "Dynamic modeling and control of soybean meal drying in a direct rotary dryer" Food and bioproducts processing, Vol. 88,
No. 2, pp 90-98,2010.

(8)M. Z. D. Abad, and S. M. Mashhadi: "Design and implementation for temp-weight and humidity control of dryer based on fuzzy logic", Control, Instrumentation and Automation (ICCIA), 2011 2nd International Conference on IEEE, pp 463-467,2011.

(9)N. Behroozi Khazaei,T. Tavakoli,H. Ghassemian,M. H. Khoshtaghaza, and A. Banakar: "Applied machine vision and artificial neural network for modeling and controlling of the grape drying process", Computers and Electronics in Agriculture, No. 98, pp 205-213,2013.

(10) J. Ho;S. Chou,A. Mujumdar,M. Hawlader, and K. Chua: "An optimisation framework for drying of heat-sensitive products", Applied thermal engineering, Vol. 21, No. 17, pp1779-1798, 2001.

(11) J. Tao: " The design and application of an supervision system among different minitype PLCs Based on CAN-bus",Soochow University, 2008.

(12)Y. Luo,X. Jiao,W. Ji,C. Zhou,L. Zhang, and T. Li: "Control Network Communication for Pipeline Welding Based on CANopen", Procedia Engineering, No. 15, pp 4994-4999,2011. 